\title{
The Effects of the Amount of Bladder Filling on Normal Tissue Doses in 3-dimensional HDR Vaginal Cuff Brachy Therapy
}

\author{
(1) Illhami ER, ${ }^{1}$ (D) Şeyda KINAY,' (D) Recep KANDEMIR, ${ }^{2}$ (D Funda OBUZ, ${ }^{3}$ (D) Ayşe Nur DEMIRAL' \\ 'Department of Radiation Oncology, Dokuz Eylül University Faculty of Medicine, İzmir-Turkey \\ 2Department of Medical Physics, Dokuz Eylül University Institute of Health Sciences, İzmir-Turkey \\ ${ }^{3}$ Department of Radiology, Dokuz Eylül University Faculty of Medicine, İzmir-Turkey
}

\begin{abstract}
OBJECTIVE
This study aimed to compare the dose-volume parameters of organs at risk (OAR) (bladder, rectum, sigmoid and bowel) for the bladder filling of 50cc versus $150 \mathrm{cc}$ in high-dose-rate (HDR) (3-D) vaginal cuff (VC) brachytherapy (BRT).

\section{METHODS}

The treatment data of eight hysterectomized patients with gynecological malignancy who received postoperative pelvic external radiotherapy (RT)+3-D HDR VC-BRT were analyzed. Recontouring was performed on CT images, and BRT plans were reformed. Proximal 1/3 vagina was treated in all patients using a cylinder. A total dose of $18 \mathrm{~Gy}$ ( $3 \times 6 \mathrm{~Gy})$ was given $(0.5 \mathrm{~cm}$ depth from the vaginal mucosa). OAR and clinical target volume (CTV) were recontoured in a 3-D manner. Afterwards, treatment planning was performed by a medical physicist using the BRT treatment planning system. Bladder V50\%, D50\% and D2cc, rectum D2cc, sigmoid D2cc, bowel D50\% and D2cc were recorded from the dose-volume histograms obtained in the treatment planning system. Paired comparisons were made for the parameters above for the bladder filling of $50 \mathrm{cc}$ versus $150 \mathrm{cc}$. Two different amounts of bladder filling were compared using the Wilcoxon Signed-Rank Test in the SPSS 15.0 statistics program.
\end{abstract}

\section{RESULTS}

Bladder D50\% decreased ( $p=0.012)$ while bladder D2cc increased $(\mathrm{p}=0.025)$ in the case of $150 \mathrm{cc}$ bladder filling instead of 50cc. Rectum D2cc showed a statistical trend for increase ( $\mathrm{p}=0.05)$; however, bowel $\mathrm{D} 50 \%$ decreased $(\mathrm{p}=0.012)$ in $150 \mathrm{cc}$ bladder filling compared to $50 \mathrm{cc}$.

\section{CONCLUSION}

The statistically significant decrease in bladder and especially bowel D50\% parameters supports filling the bladder with $150 \mathrm{cc}$ instead of 50cc in 3-D VC-BRT.

Keywords: Bladder filling; normal tissue dose; 3-dimensional vaginal cuff brachytherapy. Copyright $\odot$ 2019, Turkish Society for Radiation Oncology

\section{Introduction}

Three-dimensional (3-D) brachytherapy (BRT) has been increasingly used in gynecologic tumors recently. A relevant study in the literature reported that comparative dosimetric evaluation of $2 \mathrm{D}$ and $3 \mathrm{D}$ CT- based treatment planning for the same brachytherapy session demonstrates underestimation of OAR doses and the overestimation of target coverage in $2 \mathrm{D}$ treatment planning.[1] 3-D BRT planning has the potential to increase local control and reduce the side effects of critical organs, such as the rectum, sigmoid, bowel and 
bladder. Using bladder filling (BF), this study aimed to move bowel away from the high-dose region in gynecologic 3-D BRT since bowel is more sensitive than sigmoid and rectum.

There is sufficient knowledge about the potential effects of BF on OAR doses in BRT in definitive RT of cervix cancer. However, few studies exist examining the effects of BF on OAR doses in vaginal cuff (VC) BRT where bowels fall into the pelvis due to the removal of the uterus, [2-6] which remained under-researched.

According to the recommendations of the European Society for Radiotherapy and Oncology-European Brachytherapy Group (GEC-ESTRO),[7] the bladder is filled with an amount of $50 \mathrm{cc}$. In our study, $50 \mathrm{cc}$ was compared to $150 \mathrm{cc} \mathrm{BF}$ (a value higher than $100 \mathrm{cc}$ recommended by Hoskin et al.[5]) in patients receiving 3-D VC-BRT after postoperative external pelvic RT. It is important to note that, unlike the comparisons of the empty versus filled bladder performed in the previous studies, to our knowledge, our study is the first study comparing different $\mathrm{BF}$ quantities to find out if $150 \mathrm{cc}$ is more advantageous regarding OAR.

In our study, dose-volume parameters (DVPs) of OAR (volume receiving $50 \%$ of the dose (V50\%), dose received by $50 \%$ of the volume (D50\%), minimum dose for the $2 \mathrm{~cm}^{3}$ volume receiving the highest dose (D2cc) for bladder; D2cc for rectum and sigmoid; D50\% and $\mathrm{D} 2 \mathrm{cc}$ for bowel) were compared in the case of $\mathrm{BF}$ of 50 cc and $150 \mathrm{cc}$.

\section{Materials and Methods}

In this study, the study population was identified as hysterectomized patients with a diagnosis of gynecological malignancy receiving postoperative pelvic ERT $+3-\mathrm{D}$ HDR VC-BRT in the Department of Radiation Oncology in Dokuz Eylul University Medical Faculty between March and August 2015. Retrospectively, CT sectional images were used for this study; recontouring was performed, and BRT plans were formed again. Ethical approval was obtained for this study from Dokuz Eylul University Ethics Committee for Non-invasive Research (Decision Number: 2015/21-14).

Pelvic RT was planned with the Eclipse v11 TP system and was delivered with TrueBeam STx high-energy linear accelerator. The diagnosis was endometrium cancer in five, and cervix cancer in three patients. In intracavitary BRT, a CT-compatible plastic cylinder applicator (stump applicator, GM1 1004160) was used.

For the optimal coverage of clinical target volume (CTV), a stump applicator (SA) with the tolerable larg- est diameter was preferred. SA diameter was 2.6-3.5 $\mathrm{cm}$. The preferred SA in the first application was used in the subsequent applications. After placement, the applicator was immobilized by attaching it to a rigid external fixator on the BRT bed. Proximal 1/3 vagina was treated in all patients. HDR BRT dose was administered and the Ir-192 source had been prescribed to $0.5 \mathrm{~cm}$ depth from the vaginal mucosa (SA surface). A total dose (TD) of 18 Gy was performed with a fraction dose of 6 Gy administered once or twice a week.

All patients were ordered to drink laxatives a night before the application and not to eat anything so that rectum, sigmoid, and bowels could stay in the same position with minimum fullness. First, a foley catheter (FC) was placed and fixed by filling the catheter balloon with $7 \mathrm{cc}$ contrast material. According to the current BRT imaging protocol in our clinic, CT-scan was performed at two different amounts of $\mathrm{BF}$ ( 50 and $150 \mathrm{cc}$ ), and $150 \mathrm{cc}$ filling was preferred in the presence of a significant visual difference in removal of the bowels away from the applicator. First, $50 \mathrm{cc}$ saline was administered after emptying bladder through an FC; then, the catheter was clamped. After the placement of the applicator, the patient underwent the first scan in the CT-simulator device (Somatom Definition AS). A second CT-scan was performed immediately in the same position after clamping the catheter following additional BF with $100 \mathrm{cc}$ saline. The patients did not experience any discomfort in the case of $150 \mathrm{cc}$ bladder filling. During CT-scan and BRT, the patient's position was the same (supine position, legs placed on supports and knees slightly twisted). Applicator was located in the midline of the patient, parallel to the ground. Applicator position was checked using AP and lateral Xrays before $\mathrm{CT}$-scan.

During CT-scan, no oral or intravenous contrast material was administered, and $3 \mathrm{~mm}$ slice thickness was used. On CT images, it was confirmed that the applicator was in contact with the VC apex. CT images were transferred to the computerized TP system (Brachy Vision Brachytherapy TP System). After CTV and OAR contours were completed, the BRT planning was performed. The most appropriate BRT plan was selected and was applied using the GammaMedplus iX HDR remote-afterloading BRT device.

Bladder, rectum, sigmoid, and bowel were determined as OAR in our study. OAR and CTV were contoured retrospectively by a radiation oncology resident. These contours were first controlled by a staff radiation oncology and radiology lecturer, respectively. 
A specific contouring protocol was established: 1) Bladder was contoured as the whole organ. 2) Rectum was defined as the bowel segment extending from the beginning of sigmoid to the lower part of femoral heads. 3) Sigmoid was defined as the portion extending from the end of the rectum to the junction where the bowel converts from transverse to vertical position. 4) Since oral contrast is not routinely used in our daily practice for vaginal cuff brachytherapy because of patient discomfort and workload, it was impossible to differentiate small bowel from the large bowel. Thus, the bowel was defined as small and large intestine segments that extend to the lower level of the sacroiliac joint. Inferiorly bowel was contoured from the most inferior small or large bowel loop. Muscle and bones, as well as any overlapping non-gastrointestinal normal structures, were excluded. Considering the high mobility of bowel, the bowel was contoured in the form of a "bowel bag" with the surrounding omentum instead of "bowel loops".

After completion of the contouring process, planning was made by a medical physicist using the BRT TP system. For CTV, values for D100\%, D90\%, D15\%, D5\% parameters were defined. For rectum, sigmoid, and bladder, D2cc dose limits were defined. BRT planning was performed considering the ERT+BRT TD limitations of OAR D2cc ( $<90$ Gy for bladder, $<70-75$ Gy for rectum and sigmoid) regarding equivalent dose with 2 Gy/fraction (EQD2) according to $\alpha / \beta: 3$ as recommended by GEC-ESTRO.[7,8] It was checked for both BF amounts whether the CTV parameters (D100\% $\geq 70 \%$, D $98 \% \geq 85 \%$, D $90 \% \geq 105 \%$, V100\% $\geq 92.5 \%$ and D50\%/ D $90 \% \leq 1.5$ ) were met. In our study, OAR DVPs (V50\%, $\mathrm{D} 50 \%, \mathrm{D} 2 \mathrm{cc}$ for the bladder, D2cc for rectum and sigmoid, D50\% and D2cc for bowel) were compared in two BF amounts. Statistical analysis of the relevant parameters was performed using the SPSS 15.0 statistical program. Wilcoxon Signed-Rank Test was used to compare two different BF quantities since the nonparametric test was required due to the study population being less than 30 .

\section{Results}

\section{Patient Characteristics}

Patient, tumor, and treatment characteristics are shown in Table 1.

\section{Calculated Bladder Volumes}

Mean bladder volumes were 136 (71-261) cc and 244.8 (158-350) cc for 50 and $150 \mathrm{cc}$ BF, respectively. Over-
Table 1 Patient, tumor and treatment characteristics

\begin{tabular}{|c|c|}
\hline Characteristic & $\begin{array}{c}\text { Number of the } \\
\text { patients }\end{array}$ \\
\hline \multicolumn{2}{|l|}{ Age, years } \\
\hline$\leq 60$ & 6 \\
\hline$>60$ & 2 \\
\hline \multicolumn{2}{|l|}{ Diagnosis } \\
\hline Endometrium carcinoma & 5 \\
\hline Cervix carcinoma & 3 \\
\hline \multicolumn{2}{|l|}{ FIGO Stage } \\
\hline \multicolumn{2}{|l|}{ Endometrium carcinoma } \\
\hline Stage I & 2 \\
\hline Stage II & 2 \\
\hline Stage III & 1 \\
\hline \multicolumn{2}{|l|}{ Cervix carcinoma } \\
\hline Stage I & 2 \\
\hline Stage III & 1 \\
\hline \multicolumn{2}{|l|}{ Histology } \\
\hline \multicolumn{2}{|l|}{ Endometrium carcinoma } \\
\hline Adenocarcinoma & 5 \\
\hline \multicolumn{2}{|l|}{ Cervix carcinoma } \\
\hline Adenocarcinoma & 1 \\
\hline Squamous cell carcinoma & 2 \\
\hline \multicolumn{2}{|l|}{ Tumor Grade } \\
\hline \multicolumn{2}{|l|}{ Endometrium carcinoma } \\
\hline Grade 1 & 4 \\
\hline Grade 2 & 1 \\
\hline \multicolumn{2}{|l|}{ Cervix carcinoma } \\
\hline Grade 2 & 2 \\
\hline Unknown & 1 \\
\hline \multicolumn{2}{|l|}{ Treatment schedule } \\
\hline 45 Gy pelvic ERT + 18 Gy BRT & 7 \\
\hline 45 Gy pelvic + paraaortik ERT + 18 Gy BRT & 1 \\
\hline \multicolumn{2}{|l|}{ Cylinder diameter (cm) } \\
\hline 2.6 & 1 \\
\hline 3.5 & 7 \\
\hline
\end{tabular}

ERT: External radiotherapy; BRT: Brachytherapyi.

all, it was observed that an increase in BF pushes the bowels upward and away from the applicator.

\section{Target Volume}

CTV DVPs from BRT TP with two different amounts of BF are shown in Table 2.

\section{Organs at Risk}

When the OAR DVPs from ERT and BRT TP were examined, bladder D2cc ERT+BRT total EQD2 (mean \pm st. error) was 67.8 \pm 1.1 (63.7-73.0) Gy and 71.1 \pm 0.8 (66.374.5) Gy with 50 and $150 \mathrm{cc} B F$, respectively. Sigmoid D2cc ERT+BRT total EQD2 was 57.6 \pm 2.7 (45.2-66.2) 
Table 2 CTV dose-volume parameters in 50 and $150 \mathrm{cc}$ bladder filling

\begin{tabular}{lcc} 
& $\begin{array}{c}\mathbf{5 0} \text { cc bladder filling } \\
\text { Mean } \pm \text { st. error }\end{array}$ & $\begin{array}{c}\mathbf{1 5 0} \text { cc bladder filling } \\
\text { Mean } \pm \text { st. error }\end{array}$ \\
\hline D100\% & $85.1 \pm 1.5$ & $85.6 \pm 1.8$ \\
D98\% & $99.6 \pm 1.0$ & $100.1 \pm 1.2$ \\
D90\% & $107 \pm 0.8$ & $107.9 \pm 1.1$ \\
D50\% & $127.4 \pm 1.1$ & $128.3 \pm 1.2$ \\
D50\%/D90\% & $1.2 \pm 0.01$ & $1.2 \pm 0.01$ \\
V100\% & $97.5 \pm 0.5$ & $97.7 \pm 0.6$ \\
V150\% & $16.3 \pm 1.8$ & $17.3 \pm 2.1$ \\
\hline
\end{tabular}

Gy and 58.3 \pm 3.3 (44.5-70.4) Gy with 50 and $150 \mathrm{cc}$ BF, respectively. Rectum D2cc ERT+BRT total EQD2 was 63.7 \pm 2.7 (50.2-72.6) Gy and 64.4 \pm 2.5 (50.8-71.5) Gy with 50 and $150 \mathrm{cc} \mathrm{BF}$, respectively.

\section{Bladder}

Comparison of the bladder DVPs with two different amounts of BF is shown in Table 3. Bladder V50\% was reduced if the bladder was filled $150 \mathrm{cc}$ instead of 50cc. This decrease showed a trend toward significance $(\mathrm{p}=0.069)$. Bladder D2cc increased significantly $(\mathrm{p}=0.025)$, while $\mathrm{D} 50 \%$ decreased significantly $(\mathrm{p}=0.012)$ when the bladder was filled $150 \mathrm{cc}$.

\section{Rectum and Sigmoid}

Table 4 shows the comparison of the rectum and sigmoid DVPs with two different amounts of BF. Rectum D2cc was increased when bladder was filled $150 \mathrm{cc}$ instead of $50 \mathrm{cc}$. However, this increase was of borderline significance $(\mathrm{p}=0.05)$. On the other hand, sigmoid $\mathrm{D} 2 \mathrm{cc}$ was similar for two different amounts of BF.

\section{Bowel}

Comparison of bowel DVPs with two different amounts of $\mathrm{BF}$ is shown in Table 5. Bowel D2cc was similar in the case of $50 \mathrm{cc}$ and $150 \mathrm{cc}$ filling. Bowel D50\% was significantly decreased $(\mathrm{p}=0.012)$ when the bladder was filled $150 \mathrm{cc}$. Figure 1 shows the proportional reduction of bowel volume entering the medium-high dose region by filling bladder $150 \mathrm{cc}$ instead of $50 \mathrm{cc}$.

Table 3 Comparison of the bladder dose-volume parameters in two different amounts of bladder filling

\begin{tabular}{|c|c|c|c|}
\hline Bladder dose-volume parameters & $\begin{array}{c}50 \text { cc bladder filling } \\
\text { Mean } \pm \text { st. error } \\
\text { Median ( } 25 \%-75 \% \text { quartile) } \\
\text { Range }\end{array}$ & $\begin{array}{c}150 \text { cc bladder filling } \\
\text { Mean } \pm \text { st. error } \\
\text { Median ( } 25 \%-75 \% \text { quartile) } \\
\text { Range }\end{array}$ & $\mathbf{p}$ \\
\hline \multirow[t]{3}{*}{ D2cc (Gy) } & $14.1 \pm 0.4$ & $15.4 \pm 0.3$ & 0.025 \\
\hline & $14.1(12.9-14.9)$ & $15.5(15.1-15.9)$ & \\
\hline & $12.5-16.1$ & $13.5-16.7$ & \\
\hline \multirow[t]{3}{*}{ V50\% (\%) } & $17.9 \pm 3.2$ & $14.7 \pm 2.3$ & 0.069 \\
\hline & $15.7(9.5-27.3)$ & $14.3(9.6-20.6)$ & \\
\hline & 7.3-31.9 & $5.5-24.9$ & \\
\hline \multirow[t]{3}{*}{ D50\% (Gy) } & $5.4 \pm 0.5$ & $4.5 \pm 0.5$ & 0.012 \\
\hline & $5.3(4.2-6.8)$ & $4.6(3-5.7)$ & \\
\hline & 3.2-7.3 & 2.6-6.3 & \\
\hline
\end{tabular}

Table 4 Comparison of the rectum/sigmoid dose-volume parameters in two different amounts of bladder filling

Rectum/sigmoid dose-volume parameters

Sigmoid D2cc (Gy)

Rektum D2cc (Gy)
50 cc bladder filling

Mean \pm st. error Median (25\%-75\% quartile)

Range

$12.1 \pm 1.2$

$12.2(10.4-14.9)$

5.6-15.9

$9.8 \pm 1.2$

$11.3(5.7-12.7)$

$5.5-13.8$
150 cc bladder filling

Mean \pm st. error

Median (25\%-75\% quartile)

Range

$12.5 \pm 1.1$

0.05

$13(11.2-15.0)$

$6.1-15.5$

$10 \pm 1.5$

0.779

11.0 (5.7-13.7)

4.8-15.5 
Table 5 Comparison of the bowel dose-volume parameters in two different amounts of bladder filling

Bowel dose-volume parameters

Mean \pm st. error
Median (25\%-75\% quartile)
Range

$16.3 \pm 1.6$

$17.3(16.2-18.8)$

5.8-19.6

D50\% (Gy)
$2.6 \pm 0.3$

$2.8(2.2-3.2)$

$0.7-3.3$
150 cc bladder filling

Mean \pm st. error

Median (25\%-75\% quartile)

Range

$14.0 \pm 1.5$

0.123

$14.6(9.7-17.1)$

7.9-19.2

$2.2 \pm 0.3$

$2.2(1.7-2.9)$

$0.7-3$

\section{Discussion}

\section{Application}

In some of the studies, we noticed that the filling method was not reliable enough since patients were not catheterized during application. [2,4] In these studies, it was seen that the variability in bladder volumes reached up to 13.4 and 16 times among patients in the case of the empty and full bladder, respectively. $[2,4]$ In other studies, as in ours, BF was achieved by giving a certain amount of fluid through $\mathrm{FC}$ and ensuring more reliable and standardized BF.[3,5,6] Calculated bladder volumes were reported in only one[3] of two studies $[3,6]$ performed in the era of 3-D BRT. In the study of Hung et al.,[3] variability in bladder volumes was only 2.9 and 1.8 times among patients in the case of the empty and full bladder $(180 \mathrm{cc})$, respectively. Similarly, in our study, the ratio of maximum/minimum value of bladder volumes was only 3.7 and 2.2 times among patients for $50 \mathrm{cc}$ and $150 \mathrm{cc}$ filling, respectively. Variability in volume was minimized by direct delivery of the fluid to the bladder with FC.

We observed that bladder volume calculated by the TP system was not below $50 \mathrm{cc}$ in patients with BF of $50 \mathrm{cc}$, and was not below $150 \mathrm{cc}$ in patients with BF of 150 cc. In Hung's study, [3] minimum bladder volume was $161 \mathrm{cc}$, although the bladder was filled with $180 \mathrm{cc}$ via FC. This can be explained by the backward flowing of liquid at the time of catheter clamping after BF. In our study, calculated bladder volumes were found to reach up to 5.2 and 2.3 times the targeted volume values for $50 \mathrm{cc}$ and $150 \mathrm{cc} \mathrm{BF}$, respectively. Similarly, in Hung's study, [3] calculated bladder volume reached to a maximum of 1.6 times of the targeted one for 180 cc filling. Despite filling bladder with the same amount of liquid in all patients in both studies, reasons of the variability in bladder volume among patients may be related to the following: Variability in liquid intake and loss within 24-hour period before the BRT, emptying of bladder via FC prior filling, bladder flexibility, capacity, renal clearance, and leaking of air into bladder unintentionally.

\section{Contouring}

In our study, sigmoid was defined as the part of the bowel beginning from the proximal end of the rectum and extending to the junction of vertical and transversal parts of the bowel. On the other hand, sigmoid was contoured being included in the bowel in Kobzda et al.'s study.[2] However, since tolerance doses of sigmoid and small bowel differ from each other, it was more relevant to contour these organs separately. In our study, the bowel was defined as small and large bowel segments above the lower level of sacroiliac joint except for rectum and sigmoid; these segments were contoured as "bowel bag" together with surrounding omentum. We observed that bowel was contoured as bowel loops, instead of "bowel bag" in some studies. $[2,3,6]$ Due to the absence of oral contrast during CTsimulation in our retrospective study, it was possible to distinguish small and large bowel clearly. Additionally, bowel segments may be displaced intra- and interfractionally during BRT. Thus, contouring bowels as a "bowel bag" was considered to enable bowel DVPs to be evaluated more accurately. It was detected that only $20 \%$ of bowels kept the same position during the whole period of treatment in patients receiving pelvic IMRT.[9]

\section{Treatment Planning}

\section{Target Volume}

In our study, D100\%, D98\%, D90\%, V100\%, and D50\%/D90\% parameters of CTV were met in each patient for both $50 \mathrm{cc}$ and $150 \mathrm{cc} \mathrm{BF}$. It is crucial to assure 
that BRT treatment plans with two different amounts of $\mathrm{BF}$ compete under the same conditions regarding providing certain CTV DVPs. On the other hand, no criteria were reported for CTV DVPs in other studies.[2-6]

\section{Normal Tissues}

In our study, BRT planning was performed considering ERT+BRT TD restrictions of OAR D2cc (for bladder $<90 \mathrm{~Gy}$, for rectum and sigmoid $<70-75 \mathrm{~Gy}$ ) regarding EQD2 according to $\alpha / \beta: 3$ as recommended by GEC-ESTRO. [7,8] OAR D2cc TD restrictions were exceeded in none of our patients. Kobzda et al.[2] also observed that bladder D2cc TD did not exceed the recommended limits in any patient. In BRT, it is recommended that D50\% (OAR mean dose) also must be taken into consideration in addition to Dcc parameters $(\mathrm{D} 0.1 \mathrm{cc}, \mathrm{D} 1 \mathrm{cc}, \mathrm{D} 2 \mathrm{cc})$ representing hot spots as being the most frequently used for sigmoid, rectum, and bladder.[10]

\section{Bladder}

In our study, V50\% showed a trend to decrease in 150 cc BF compared to $50 \mathrm{cc}$. While D50\% decreased, D2cc increased, both being significant. In Kobzda et al.'s study, bladder DVPs were compared in empty versus filled bladder (obtained with $400 \mathrm{cc}$ water intake 40 minutes before CT- scan).[2] Their findings showed that bladder V50\% and V80\% were significantly lower and bladder D2cc and D0.1cc were significantly higher in the filled bladder.[2] In the study of Hung et al., bladder D2cc was shown to have a tendency to increase, and bladder D50\% decreased significantly for the BF of $180 \mathrm{cc}$ versus empty bladder.[3] In Stewart et al.'s study, bladder DVPs were compared in empty versus filled bladder (obtained with $946 \mathrm{cc}$ water intake 1 hour before CT-scan).[4] Bladder V70\%, V50\% and D2cc were observed significantly higher in the filled bladder. In Hoskin and Vidler's study performed using CT but without $\mathrm{DVH}$, no significant variability in bladder doses was observed with BF amounts of 35 , 70 and $100 \mathrm{cc}$.[5] In the study of Güler et al., bladder DVPs were compared in empty versus $180 \mathrm{cc}$ filled bladder.[6] Bladder D2cc increased without a significant change in bladder D50\%.

Except for one study,[3] all studies, including ours, $[2,4,6]$ have demonstrated a significant increase in bladder D2cc as the bladder is filled. Since bladder D2cc is kept within ERT+BRT TD restriction, this issue does not have considerable clinical importance. The reason for the increase in bladder D2cc may be the approaching of the bladder portion adjacent to $\mathrm{VC}$ to the BRT high-dose region due to gravitation in a supine position. However, it should be noted that BF more than $150 \mathrm{cc}$ causes the approaching of the posterior wall of the bladder to cylinder to a greater extent and thus, may cause exceeding of bladder D2cc TD limit. There are two studies of VC-BRT evaluating bladder D50\%.[3,6] Similar to our study, a significant decrease with an increase in $\mathrm{BF}$ was observed in one [3] of them. In one[2] of the two studies[2,4] evaluating bladder V50\% in VC-BRT, a decrease was noted when the bladder fills, as in our study. It is thought that this situation is related to the decrease of the ratio of bladder volume approaching BRT medium-high dose region to total bladder volume as $\mathrm{BF}$ increases. Although ERT+BRT TD limits are not as clear as for Dcc parameters, the significant decrease of bladder D50\% supports $150 \mathrm{cc} \mathrm{BF}$.

\section{Rectum-Sigmoid}

In our study, rectum D2cc increased with $150 \mathrm{cc} \mathrm{BF}$ compared to $50 \mathrm{cc}$. although this increase was of borderline significance $(p=0.05)$. None of the existing studies in VC-BRT show any change in rectum D2cc with BF. [2-4,6] In our study, sigmoid D2cc was similar for the two amounts of BF. Also, no change in sigmoid $\mathrm{D} 2 \mathrm{cc}$ was observed with $\mathrm{BF}$ in the two studies with sigmoid contouring. [3,6] It is thought that rectum and sigmoid doses are not affected by BF because these organs are anatomically located posteriorly and relatively distant from the bladder.

\section{Bowel}

In our study, bowel D2cc was similar for 50 and $150 \mathrm{cc}$ amounts of BF. However, bowel D50\% decreases significantly in the case of $150 \mathrm{cc} B F$. By increasing BF, bowel D2cc decreased only in one study,[3] but it did not change in the other studies[2,6] in accordance with our results. The reason for the bowel D2cc decrease not being significant in our study may be because of the contouring of bowels as a "bowel bag". In the solitary study evaluating bowel D50\%,[3] a significant decrease was observed with increasing BF, similar to our results. In the study of Hoskin and Vidler, $57.5 \%$ decrease was detected in the amount of small bowel inside the highdose region in $100 \mathrm{cc}$ BF compared to the empty bladder.[5] In our study, bowel D50\% showed a mean decrease of $18.3 \%$ with increasing BF. It is suggested that this situation is related to the decrease in the amount of bowel approaching BRT medium-high dose region due to the pushing of the bowel cranially by the filled bladder (Fig. 1). 


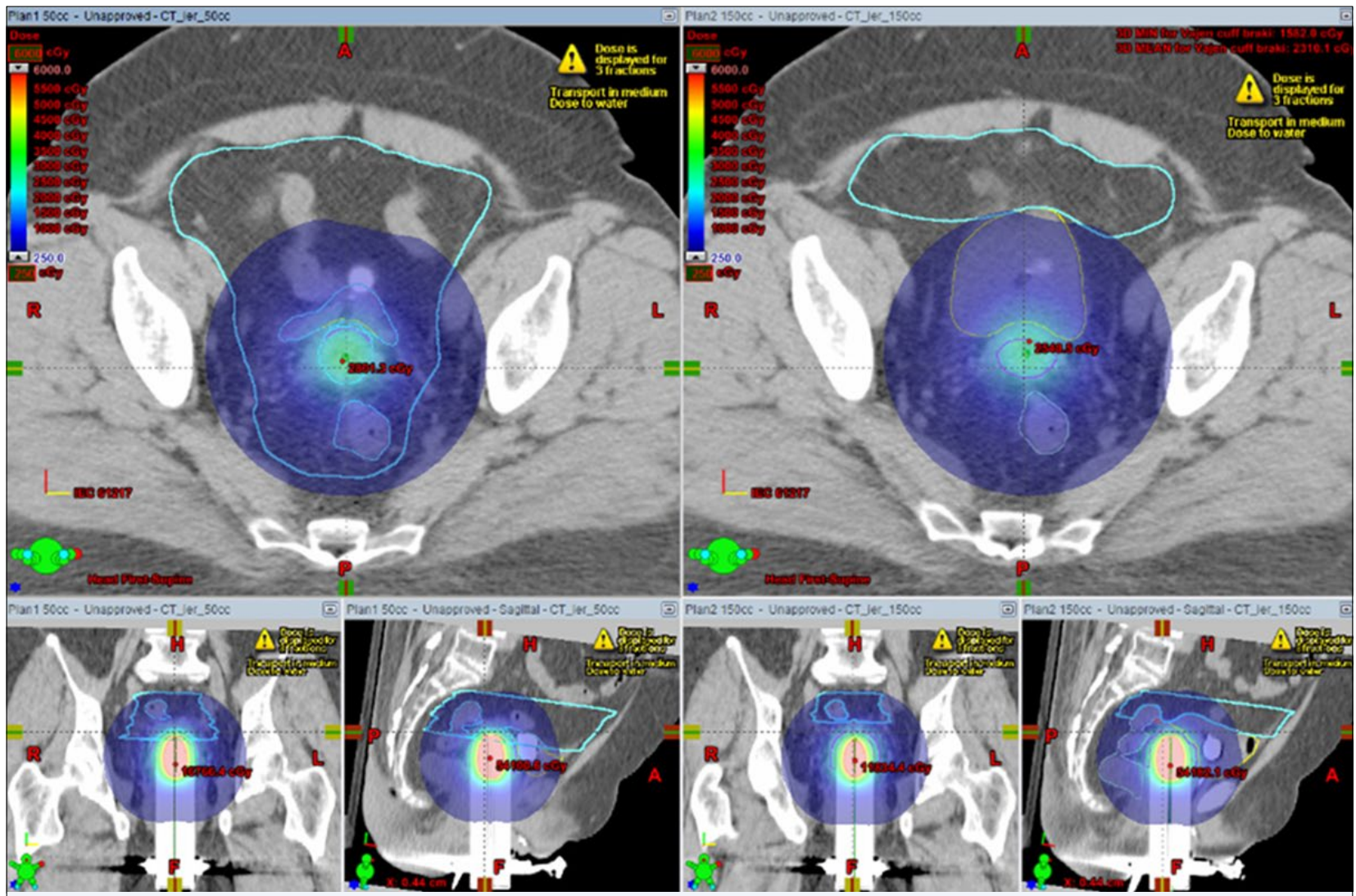

Fig. 1. (a) $(50 \mathrm{cc})$ and (b) $(150 \mathrm{cc})$ Proportional reduction of the bowel volume in mid-high dose region with increase in bladder filling (2.5 Gy isodose).

\section{Limitations of this Study}

The limitation of this study is the small number of patients. As in most of the previous studies, the variability in the realized amount of the BF although using FC is another problematic issue. This variability can be reduced using ultrasound both just before CT-simulation and BRT sessions to control the emptiness of the bladder before filling procedure. Despite the limitations of this study, it seems that findings support $150 \mathrm{cc}$ BF in 3-D VC-BRT.

\section{Conclusion}

Present studies have compared empty with filled bladder; however, to our knowledge, our study is the first study comparing two different amounts of BF (50 cc and $150 \mathrm{cc}$ ) regarding OAR sparing. A significant decrease in bladder D50\% supports $150 \mathrm{cc}$ filling. Also, for bowels with the highest risk of RT toxicity, $150 \mathrm{cc}$ filling seems more advantageous than $50 \mathrm{cc}$ in the light of the significant decrease in bowel D50\%.
Peer-review: Externally peer-reviewed.

Conflict of Interest: None declared.

Financial Support: None declared.

Authorship contributions: Concept - İ.E., A.N.D.; Design - İ.E., Ş.K., R.K., F.O., A.N.D.; Supervision - A.N.D.; Materials - İ.E., A.N.D.; Data collection \&/or processing - İ.E., Ş.K., R.K., F.O., A.N.D.; Analysis and/or interpretation - İ.E., Ş.K., R.K., A.N.D.; Literature search - İ.E., A.N.D.; Writing - İ.E., Ş.K., R.K., F.O., A.N.D.; Critical review - İ.E., Ş.K., R.K., F.O., A.N.D.

\section{References}

1. Madan R, Pathy S, Subramani V, Sharma S, Mohanti $\mathrm{BK}$, Chander S, et al. Comparative evaluation of twodimensional radiography and three dimensional computed tomography based dose-volume parameters for high-dose-rate intracavitary brachytherapy of cervical cancer: a prospective study. Asian Pac J Cancer Prev 2014;15(11):4717-21.

2. Kobzda JD, Cikowska-Wozniak E, Michalska M, Makarewicz R. Three-dimensional dosimetry of the full and 
empty bladder in high dose rate vaginal cuff brachytherapy. Int J Gynecol Cancer 2014;24(5):923-7.

3. Hung J, Shen S, De Los Santos JF, Kim RY. Imagebased $3 \mathrm{D}$ treatment planning for vaginal cylinder brachytherapy: dosimetric effects of bladder filling on organs at risk. Int J Radiat Oncol Biol Phys 2012;83(3):980-5.

4. Stewart AJ, Cormack RA, Lee H, Xiong L, Hansen JL, O'Farrell DA, et al. Prospective clinical trial of bladder filling and three-dimensional dosimetry in high-doserate vaginal cuff brachytherapy. Int J Radiat Oncol Biol Phys 2008;72(3):843-8.

5. Hoskin PJ, Vidler K. Vaginal vault brachytherapy: The effect of varying bladder volumes on normal tissue dosimetry. Br J Radiol 2000;73(872):864-6.

6. Guler OC, Onal C, Acibuci I. Effects of bladder distension on dose distribution of vaginal vault brachytherapy in patients with endometrial cancer. J Contemp Brachytherapy 2015;6(4): 371-6.

7. Pötter R, Haie-Meder C, Van Limbergen E, Barillot I, De Brabandere M, Dimopoulos J, et al; GEC ESTRO
Working Group. Recommendations from gynaecological (GYN) GEC ESTRO working group (II): concepts and terms in 3D image-based treatment planning in cervix cancer brachytherapy-3D dose-volume parameters and aspects of 3D image-based anatomy, radiation physics, radiobiology. Radiother Oncol 2006;78(1):67-77.

8. Pötter R, Dimopoulos J, Georg P, Lang S, Waldhausl C, Wachter-Gerstner N, et al. Clinical impact of MRI assisted dose-volume adaptation and dose escalation in brachytherapy of locally advanced cervix cancer. Radiother Oncol 2007; 83(2):148-55.

9. Sanguineti G, Little M, Endres EJ, Sormani MP, Parker BC. Comparison of three strategies to delineate the bowel for whole pelvis IMRT of prostate cancer: Radiother Oncol 2008; 88(1):95-101.

10. Sun B, Yang D, Esthappan J, Garcia-Ramirez J, Price S, Mutic S, et al. Three-dimensional dose accumulation in pseudo-split-field IMRT and brachytherapy for locally advanced cervical cancer. Brachytherapy 2015;14(4):481-9. 